\title{
UN DESTIN EXCEPTIONNEL : LES ENFANTS ABANDONNÉS AU TRAVAIL (ÉVORA, 1650-1837)
}

\author{
par Laurinda ABREU
}

Le 30 novembre 1658 Andresa est abandonnée à la naissance par ses parents et recueillie par la confrérie de la Misericórdia de Évora (Portugal). Un mois plus tard, elle reçoit le baptême comme tous les enfants trouvés, dans la cathédrale de la ville, à laquelle appartient la confrérie de la Misericórdia. Le prêtre assurant les baptêmes ce jour-là, Antonio dos Santos, est inscrit comme son parrain sur le registre paroissial qui désigne Andresa comme " enfant de l'église ${ }^{1}$. La confrérie la confie à Brites Rodrigues, la nourrice qui l'élève pendant 96 mois, soit

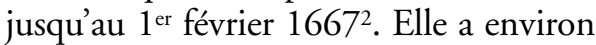
10 ans lorsque la Misericórdia rédige, le 14 juillet 1670, son premier contrat de travail. C'est le tabellion de la confrérie, Manuel Galvão Bároco, qui signe le document comme tuteur de l'enfant, et la prend à son service comme domestique. Il s'engage à l'alimenter, à l'habiller, ainsi qu'à la protéger en interdisant qu'elle sorte seule à partir de son douzième anniversaire, âge à partir duquel il commencera à lui payer 10 testons annuels qui lui seront remis à la fin du contrat. En outre, il s'engage à informer la Misericórdia dans le cas où il souhaiterait la renvoyer ; il s'engage aussi à ne jamais la confier directement à un autre tuteur ${ }^{3}$.

La raison de l'interruption du contrat de Andresa demeure inconnue. Mais l'on dispose d'un nouveau contrat, signé en décembre 1671, qui officialise sa nouvelle situation de travail après deux fugues de chez deux membres de la confrérie ${ }^{4}$. Manuel de Morais Pereira, son nouveau tuteur, répète toutes les garanties présentes dans le premier document, en particulier celle "de ne donner sa main à une autre personne qu'avec le consentement de la Misericórdia "5. Cette fois la mort du maître met un terme au contrat d'Andresa. Sans autorisation de la Misericórdia, un certain Nicolau Godinho, retire la jeune fille à la veuve de Manuel Pereira, et l'emmène chez lui. C'est là que, le 28 décembre 1674 , la confrérie va la chercher, et la confie à un autre membre qui la réclamait, le cordonnier Paulo Banha. Andresa vit treize mois chez le cordonnier mais fait une nouvelle fugue. Les dernières informations à son sujet rapportent qu'en 1675, elle vit chez le pourvoyeur de la Misericórdia, le gentilhomme Simão da Gama, également évêque de la ville. Là, elle reçoit le 26 avril 1679, les appointements correspondant au temps de travail effectué chez Paulo Banha ${ }^{6}$.

Bien que mouvementée, cette trajectoire de vie d'environ 20 ans de l'enfant trouvée Andresa représente un succès exceptionnel à plusieurs titres. Parmi les 16515 enfants abandonnés à Évora entre 1580 et 1837, Andresa appartient à l'univers très restreint des 242 enfants pour lesquels l'institution a signé des contrats de travail. Elle a résisté aux périls infectieux ainsi qu'aux possibles 
mauvais traitements des maîtres successifs qu'elle a servis, finissant par être recueillie par l'un des aristocrates les plus importants de la ville.

Andresa est aussi une exception quant à la richesse documentaire dont elle bénéficie ; habituellement les informations restent plus laconiques et moins détaillées sur les enfants trouvés d'Évora. Dispersées ça et là entre les Livros de Entradas, Livros de Despesas e pagamentos de amas dos engeitados, Livros de receita e despesa et Livros de contratos de trabalho, elles doivent trouver une petite place entre la répétition impressionnante de formules identiques, dans lesquelles ne changent que les noms des nourrices, des enfants et des maîtres, les indications de nature économique, la mention d'un quelconque événement hors du commun et, presque toujours, le couperet de la date du décès.

En recoupant les informations contenues dans les différents registres, on obtient une documentation qui permet de suivre le destin des enfants abandonnés à Évora. Notre période d'analyse débute lorsque les lacunes documentaires disparaissent, c'est-à-dire en 1650 , et s'achève lorsque la responsabilité des enfants trouvés échappe à la Misericórdia, en $1837^{7}$. $\mathrm{Au}$ cours de cette période, les représentations sociales de ces enfants ont beaucoup changé, le discours des élites en donne des indices substantiels. L'analyse de cette évolution sort du cadre de cet article, mais les changements intervenus constituent l'arrière-plan des pages qui suivent. Le principal objectif est en effet d'essayer de préciser la distance qui sépare les propos relativement utopiques des réformateurs sociaux qui ont débattu sur le sujet et la " réalité vécue ", objectivable aujourd'hui. Je propose par conséquent ici une étude de cas sur l'aristocratique et latifundiaire
Évora, résidence de la Couronne jusqu'au $\mathrm{XVI}^{\mathrm{e}}$ siècle et siège de l'évêché le plus riche du pays, dont la population comptait un peu plus de 13000 habitants intra muros (Sousa, 1979, 210) et était soumise à des changements cycliques liés aux migrations saisonnières vers la ville, à chaque printemps et à chaque automne, d'ouvriers agricoles à la recherche d'un travail dans les champs de céréales, les vignobles ou les oliveraies alentours, qui s'étendaient même vers l'Algarve.

\section{Protégés PAR LA LOI ET DOUBLEMENT ABANDONNÉS PAR LA SOCIÉTÉ : LE CAS DES ENFANTS TROUVÉS D'ÉVORA}

Dès le premier Code de lois de la période moderne au Portugal, les Ordonnances de D. Manuel I ${ }^{\mathrm{er}}$ en 1514, la protection des enfants abandonnés est réglementée.

Pour les enfants qui ne peuvent compter sur le soutien de leurs parents ou de leur famille, la loi stipule que les hôpitaux et les hospices doivent veiller à leur nourriture et à leur entretien. S'ils n'existent pas, les revenus de la municipalité, ou une taxation spécifique sur la population, s'y substituent.

Bien que la loi fût très claire quant à l'organisation de ce service d'assistance, sa mise en œuvre et son développement s'avérèrent très lents, difficiles et conflictuels. Plus que des désaccords moraux, les principaux obstacles résidaient dans le coût économique de l'assistance à ces enfants; il ralentit l'ensemble du processus. Au milieu du XVII siècle encore, cohabitaient trois façons d'assister les enfants abandonnés. L'organisation pouvait être totalement et 
directement dépendante de la municipalité, elle pouvait être placée sous la tutelle des Misericórdias (généralement lorsqu'elles avaient assuré l'administration des hôpitaux au cours de la seconde moitié du XVI ${ }^{e}$ siècle), ou bien encore, dans un grand nombre de cas, sous la responsabilité des Misericórdias mais avec un cofinancement des municipalités. Quel que soit le dispositif choisi, le modèle d'assistance est dominé par deux caractéristiques principales jusqu'à ce qu'il disparaisse au cours des années 1830 : la croissance soutenue du nombre des enfants abandonnés et les difficultés financières des institutions qui devaient veiller sur eux. Le fait que ce devoir social n'ait pas suscité d'investissement charitable suffisant fondé sur la foi, qu'il ait même été perçu comme un fardeau énorme qu'il fallait obligatoirement financer par les taxes, n'a pas rendu facile la vie de ces enfants. Sur ce plan, le cas d'Évora est tout à fait exemplaire.
La décision d'attribution de la tutelle des enfants abandonnés y fut longue et conflictuelle jusqu'à ce qu'en 1618 la confrérie de la Misericórdia finisse par affirmer son autorité sur ce service d'assistance, la maintenant jusqu'en 1837 , un an après que l'État Libéral eut décidé de suspendre l'intervention des confréries sur l'éducation de ces enfants (Abreu, 2003). Au cours de cette période, l'accroissement des abandons, non linéaire, fut néanmoins soutenu, au moins jusqu'à la crise de la décennie $1770^{8}$. Le parallélisme des courbes d'entrées et de décès indique l'ampleur de la mortalité et les chances réduites que ces enfants avaient de célébrer leur septième anniversaire (Figure 1). Le coefficient de corrélation atteint 0,81 , ce qui est très élevé dans notre domaine (Figure 2)

Si l'on considère les caractéristiques socio-économiques de la ville, le mouvement des abandons d'enfants ne paraît pas indépendant des crises économiques conjoncturelles.

Fig. 1. Total des enfants abandonnés, décédés et survivants à 7 ans. Évora 1650-1837

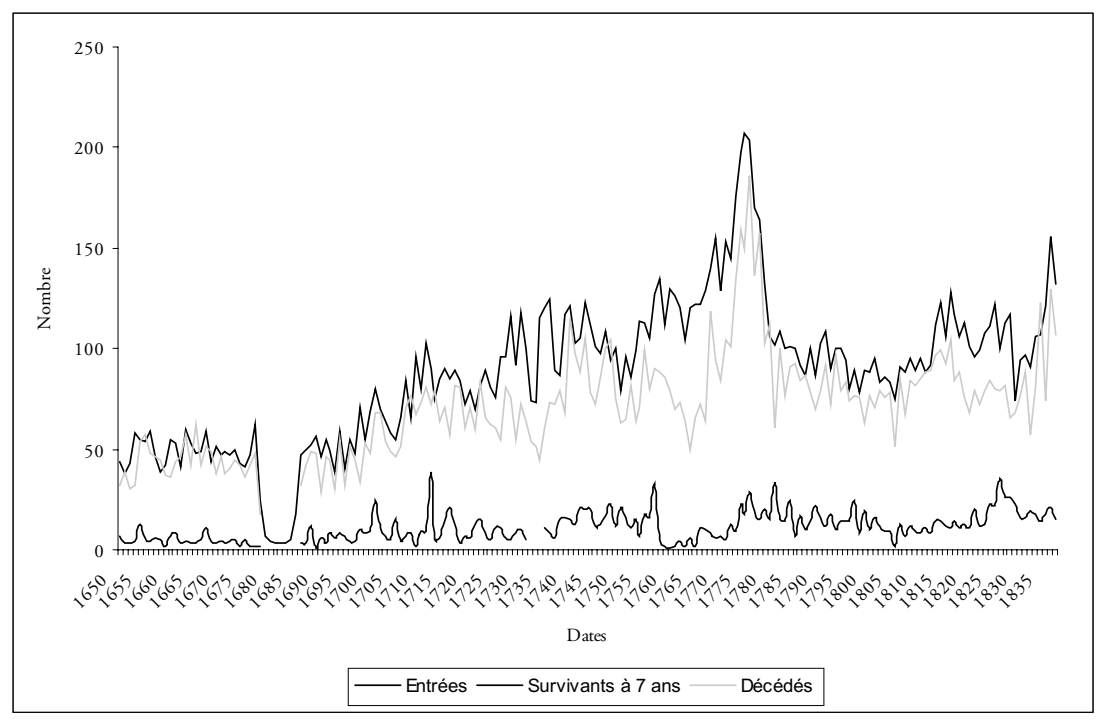


Fig. 2 La relation entre entrées des enfants et décès (droite de régression). Évora 1686-1837

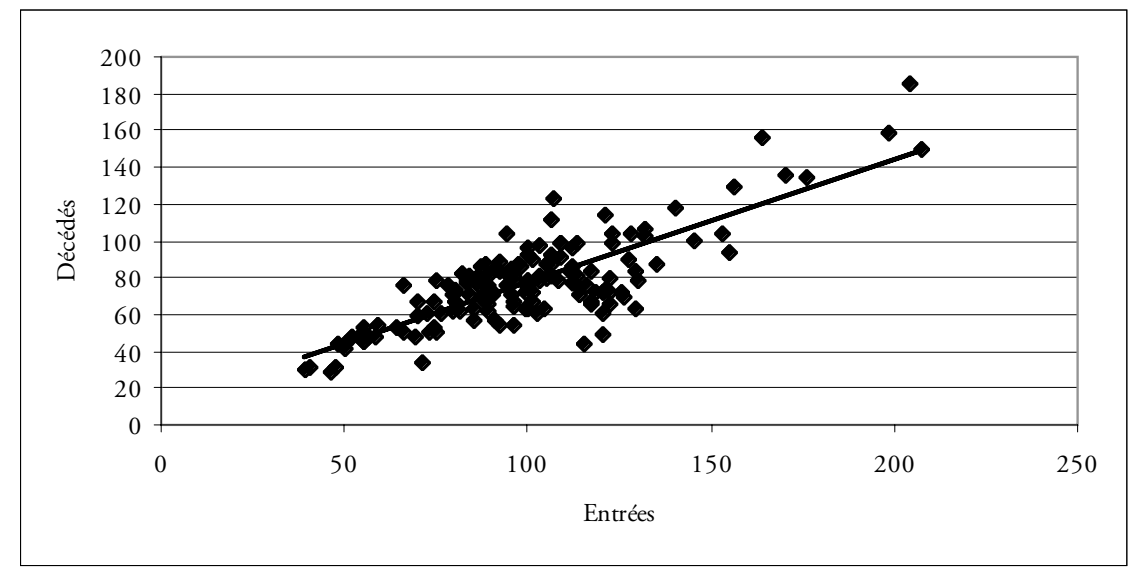

Elles ont pu s'avérer particulièrement violentes pour la population urbaine et celle de ses environs qui vivaient aux limites du seuil de subsistance, mais aussi pour celle des travailleurs migrants, qui pouvaient rester dans la région pour de fort longues périodes et y abandonner aussi leurs enfants. Même si d'autres raisons doivent être considérées dans les décisions d'abandons ${ }^{9}$, le facteur économique paraît plus déterminant que certains autres, en particulier la crainte de l'illégitimité puisque le nombre de baptêmes d'enfants illégitimes était très élevé ${ }^{10}$. Bien que certaines études aient déjà opté pour l'absence de liens, ou l'existence de liens très faibles, entre les variations de prix et celle du nombre des abandons, nous avons choisi de tester cette hypothèse à Évora car la spécificité économique de la ville, fondée sur les céréales, peut engendrer des configurations différentes.

Sur un plan général tout d'abord, le mouvement de synchronisation paraît évident, en particulier lors des cycles courts $^{11}$. La dynamisation des marchés au cours de la période d'après guerre, l'accroissement de l'offre de travail et l'augmentation de la population d'Évora qui ont pu se poursuivre jusqu'à la fin du XVII siècle n'ont pas eu d'effets très visibles sur la courbe des entrées à l'hôpital des enfants trouvés (Santos, 1995, 404). Il est certain que le nombre d'entrées s'accroît, mais lentement. En revanche, la crise sévère centrée sur l'année 1711 , qui commence par une crise céréalière " combinant de mauvaises récoltes, une situation de guerre et une pression supplémentaire afin de nourrir les armées, et une montée des prix considérable ", ne provoque pas seulement une crise démographique aiguë (Santos, 1995, 405), elle aboutit aussi à un nombre d'enfants abandonnés jamais rencontré auparavant. Plus de 100 enfants sont précisément abandonnés en 1711. Au cours des années 1715-1725, lors de la récupération de la crise, le nombre des baptêmes s'accroît ainsi que la recherche de terres. Les revenus repartent à la hausse, les contrats de travail agraires sont stabilisés et les prix des céréales décroissent. L'hôpital S. Lazaro connaît alors des années calmes, beaucoup moins d'enfants sont abandonnés. Ce cycle est bref car le phénomène se 
reproduit dans les années 1730 avec une nouvelle crise céréalière suivie par un afflux d'enfants abandonnés en particulier en 1734 et 1736 puis au cours des années 1739-1744.

La stabilisation des prix qui survient au cours de la décennie 1743-1753 correspond exactement à une diminution du nombre des abandons, même si le niveau reste élevé. Puis la décennie 17601770 ouvre une nouvelle période de crise. Tous les indicateurs se dégradent et se traduisent par une instabilité extrême des prix des céréales. La courbe de la croissance démographique s'inverse, les revenus s'affaissent et les hôpitaux croulent sous le nombre des enfants abandonnés. Pour la première fois en trois siècles d'existence, plus de 200 enfants entrent à l'hôpital S. Lazaro. Le mouvement s'amorce dès 1754 avec plus de 100 entrées, il se renforce ensuite avec 120 entrées à partir de 1764, puis, poursuivant son envol, il atteint 207 entrées en 1775. Mais si la crise économique des années 1770 est plus importante que les précédentes, il est également vrai que la récupération se révèle plus rapide et facile, du fait de la plus forte stabilité de la propriété foncière, de la plus grande efficacité et flexibilité des contrats d'affermages agricoles et dans le secteur commercial (Santos, 1995, 411).

À l'hôpital, dans la décennie des années 1780 , le nombre d'entrées redescend à son niveau du début du siècle. Après 1786 , le nombre d'enfants abandonnés passe sous la centaine, avant de retrouver ce niveau en 1811. Le nombre des entrées s'élève à nouveau lors de la grave crise céréalière de 1811-1812, dont les effets se prolongent au cours des années suivantes (entre 1813 et 1819, la moyenne annuelle d'entrées est de 115). L'économie n'enregistre plus de fortes oscillations jusqu'en
1850 , dès lors le sommet d'abandons observé en 1837 (132 entrées) doit être imputé à d'autres facteurs : l'État a simplement décidé de suspendre la tutelle des Misericórdias sur les enfants abandonnés; dans la mesure où le futur paraissait très incertain, l'inquiétude a pu conduire à des décisions d'abandons à la charité publique plus nombreux qu'en l'absence de changement de régime institutionnel.

Ce lien entre l'envolée des prix céréaliers et celle des abandons a pu être testée statistiquement pour la période de crise extrême du XVIII ${ }^{e}$ siècle, 1755-1778. Le sens de la relation entre les deux phénomènes au cours de l'envol des prix est clair : plus les prix s'élèvent et plus le nombre d'entrées à l'hôpital des enfants abandonnés monte (la pente de la courbe est particulièrement importante). En revanche, l'ajustement est de qualité moyenne (coefficient de corrélation de 0,50 ), ce qui n'est guère surprenant dans la mesure où la série des prix du blé se fonde sur des moyennes établies par année et que le total des enfants correspond aussi à des données annuelles, tandis que dans toute crise la composante saisonnière est très importante.

Cette relation entre les deux variables se retrouve lorsque la conjoncture s'inverse. Bien que le trend du nombre des abandons soit à la hausse, plus les prix baissent et plus le nombre d'entrées décline. La pente de la courbe d'ajustement est un peu moins forte que lors des années d'accroissement des prix et le coefficient de corrélation n'est que de 0,20 , mais le fait que l'on retrouve une relation entre les deux variables même lorsque la crise se résorbe plaide en faveur d'un lien systématique et global très fort.

Dans le contexte d'une économie urbaine où un pourcentage élevé de la population vit aux limites du seuil de 
Fig. 3 Relation entre le niveau des prix des céréales et le nombre des abandons. Évora 1755-1769

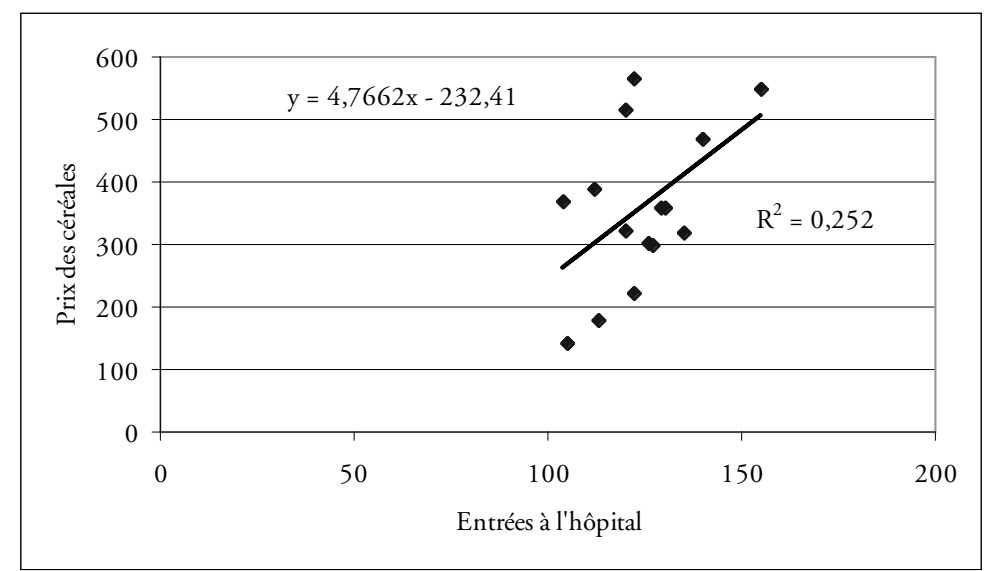

Fig. 4 Relations entre la baisse des prix céréaliers et le reflux du nombre des abandons. Évora 1769-1778

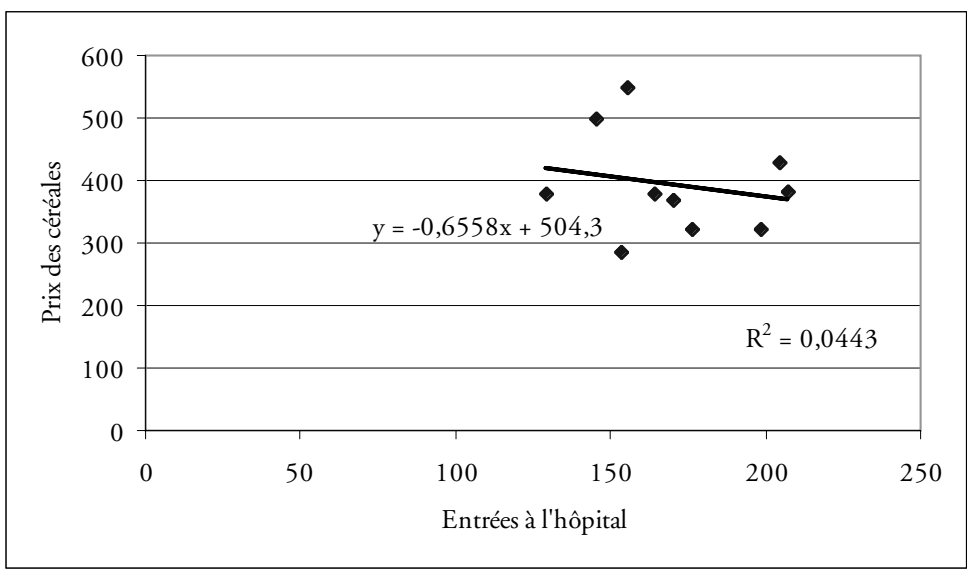

subsistance, il n'est guère surprenant qu'une crise économique se traduise immédiatement par la recherche de ressources personnelles et familiales complémentaires, ou par le désir de supprimer des dépenses. L'abandon d'un nouveau-né à l'hôpital pouvait faire partie de cet ensemble de stratégies.

Les conditions d'accueil de ces nombreux bébés avaient une conséquence majeure : de l'arrivée à l'institution jusqu'à l'âge de 7 ans, $80,5 \%$ des enfants trouvés ont été enregistrés comme morts, un chiffre qui atteint $89,5 \%$ si l'on considère que les 1485 enfants qui n'ont pas laissé de trace documentaire ont connu le même sort que les autres. La mort s'abattait particulièrement sur les plus jeunes (plus de $61,5 \%$ des enfants mouraient au cours des six premiers mois) ; si bien que la mortalité infantile s'établissait à 734 pour mille ${ }^{12}$. La dîme mortuaire s'allège ensuite, de façon habituelle, au fil des ans. La probabilité d'atteindre l'âge de 7 ans était bien faible : aux alentours de 150 pour mille (Figure 5) ${ }^{13}$. 
UN DESTIN EXCEPTIONNEL : LES ENFANTS ABANDONNÉS AU TRAVAIL (ÉVORA, 1650-1837)

Fig. 5 La courbe de survie des enfants abandonnés. Évora, 1650-1837

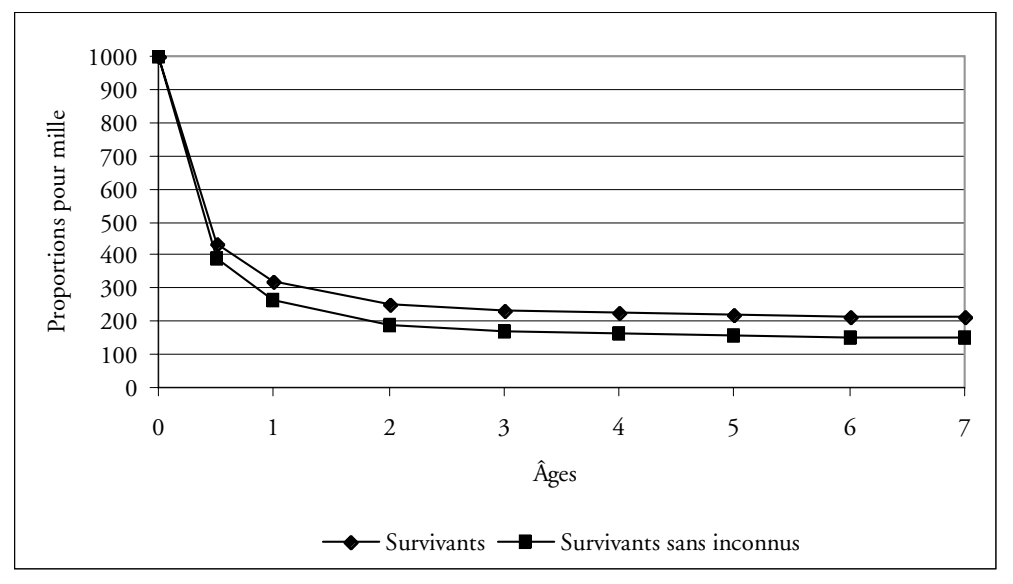

La résidence urbaine des nourrices, facteur théoriquement favorable à la survie des enfants en leur évitant les transports longs et dangereux, ne semble donc pas avoir eu d'effets très favorables. Mais il reste à préciser le temps que les enfants passaient à l'hôpital de S. Lazaro afin d'être confiés à une nourrice. Une évaluation royale réalisée en 1816 le considérait comme le facteur principal de la surmortalité des enfants trouvés ${ }^{14}$. Le même constat avait été effectué un siècle plus tôt, lorsque la nourrice officielle de l'hôpital avait été renvoyée pour négligence dans sa recherche d'autres nourrices ou pour son absence lors de l'arrivée des enfants, ce qui faisait croître le taux de mortalité15.

\section{LES SURVIVANTS : LORSQUE LA DÉNOMINATION INFLUENCE LE DESTIN}

Fauchés par la mort, omis par certains registres, banalisés par les mécanismes de dénomination, les enfants abandonnés survivants ne se laissent pas capturer dans les rets de l'historien ${ }^{16}$. Pourtant, les deux livres qui contiennent les contrats de travail, signés par la confrérie de la Misericórdia entre 1669 et 1788 pour 242 de ces enfants trouvés de plus de 7 ans, permettent de restituer quelques trajectoires. En plus des notes que les greffiers y ont consignées au fil des années lorsqu'un événement inhabituel quelconque venait rompre la monotonie des jours, ces liasses conservent la trace des dernières informations disponibles sur la plupart des enfants trouvés de la confrérie de la Misericórdia d'Évora.

Selon la loi en vigueur, la responsabilité directe des institutions qui étaient chargées des enfants abandonnés s'éteignait lorsqu'ils atteignaient leur $7^{\mathrm{e}}$ anniversaire. Le juge des orphelins devait alors les prendre en charge, c'est-à-dire leur trouver une famille d'accueil ou une place sur le marché du travail. En dépit de l'importance de ces fonctionnaires royaux, nous ne disposons d'aucune étude sur leur activité (les Collèges des orphelins sont des réalités différentes bien qu'ils aient pu intégrer des enfants trouvés), si bien qu'on ne sait pas comment ils accomplissaient 
leur charge ni avec quel taux de succès. Dans la plupart des cas, les enfants trouvés disparaissent de toute documentation dès qu'ils ont plus de 7 ans ; aujourd'hui, pour nous, une sorte de mur du silence les entoure.

Les juges n'ont probablement pas exercé régulièrement leur juridiction, soit par négligence ou parce que les confréries de Misericórdia s'y sont opposées comme ce fut le cas à Porto (Sá, 1995, 306). Dans la vaste documentation du Juge des orphelins d'Évora, on ne trouve aucun document se référant aux enfants trouvés. En revanche, les procès concernant la désignation des tuteurs et le partage de l'héritage des orphelins sont nombreux et volumineux, ce qui confirme l'affirmation de Manuel Severim de Faria quand, dans le texte portant création du collège des orphelins, il dénonçait le caractère discriminatoire des lois de protection des orphelins et des rejetés qui ne s'appliquaient en fait qu'à ceux qui disposaient d'un patrimoine, ce qui n'était pas, assurément, le cas des enfants abandonnés. On peut par conséquent supposer que l'activité des Juges des orphelins a été mobilisée par ces règlements concernant les orphelins aisés au détriment du suivi des enfants abandonnés.
Une autre confirmation de ce fait émane du témoignage de la confrérie de la Misericórdia qui, tandis qu'elle essayait de trouver des fonds pour financer l'hôpital de S. Lazaro en 1711, affirma que le manque d'argent obligeait l'institution à " expulser » les enfants trouvés dès l'âge de 7 ans sans pouvoir les former, si bien que les garçons devenaient paresseux et totalement inutiles pour la République et que les jeunes filles se trouvaient menacées par la prostitution ${ }^{17}$.

Quels furent, en définitive, les destins des 2001 enfants qui, à Évora, sont parvenus à survivre jusqu'au terme officiel de la période d'éducation ? D'emblée, constatons qu'il sont très différenciés, à l'image des renseignements existant sur chaque enfant. Pour près de $52 \%$ d'entre eux (1 037 ), le mur du silence a prévalu et nous ne disposons d'aucune information. Ils disparaissent, tout simplement, quand le greffier enregistre le dernier paiement à la nourrice. Pour les autres, nous distinguons trois grands cas : une majorité reste avec leur nourrice, certains ont été récupérés par leur famille (environ $10 \%$ de ceux pour lesquels nous disposons d'informations) et enfin $23 \%$ ont été placés sur le marché du travail par contrat.

Tab. 1 Destin des enfants abandonnés (en nombres absolus). Évora 1650-1837

\begin{tabular}{lr}
\hline Total des enfants abandonnés & 16515 \\
Total des enfants décédés & 13301 \\
Destins inconnus & 1485 \\
Survivants à 7 ans & 2001 \\
Destins inconnus au -delà de 7 ans & 1037 \\
Repris par leur famille & 113 \\
Restent chez leur nourrice & 609 \\
Sous contrat de t ravail & 242 \\
\hline
\end{tabular}


La première constatation est qu'à Évora, comme en France (BeauvaletBoutouyrie, 2003, 888 ; Brunet, Bideau, Rappalini, 2003, 912) ou en d'autres lieux, les nourrices constituaient la première option de placement des enfants après la période d'éducation (ici $60 \%$ des cas environ). Ce transfert pouvait être formalisé par la seule mention « reste chez la nourrice» ou « la nourrice l'a emmené ». Peut-être s'agissait-il d'une sorte d'adoption informelle, ou d'appoint en force de travail (quelques nourrices signaient d'ailleurs des contrats de travail), car les enfants y restaient en principe " pour la vie ». D'après quelques témoignages, ils y auraient été traités comme des membres de la famille. L'absence d'information sur de possibles rémunérations ou sur l'enseignement d'un métier laisse penser à une pleine intégration dans le cadre familial, très probablement en tant que main-d'œuvre additionnelle gratuite, mais sans acquisition des droits inhérents à l'adoption ou à la participation à l'héritage ${ }^{18}$. Aucun de ces enfants n'a fait l'objet de demande d'informations de la part de la confrérie de la Misericórdia après l'officialisation du placement définitif en dépit de la formule inscrite parfois sur le registre : « devra rendre compte au sujet de l'enfant à la Misericórdia autant de fois qu'il lui sera demandé. "C'est le silence qui domine : aucun registre de dévolution, de fugues ou de plaintes ne se retrouve parmi la documentation de la confrérie. Lautorité paternelle exercée par l'institution semble, de fait, avoir cessé avec le dernier paiement à la nourrice.

La seconde catégorie chevauche en partie la première, car les nourrices constituent aussi le groupe le plus important des familles récupérant leur enfant. Sur 113 enfants retrouvant leur famille, on ne dispose d'informations complémentaires que pour 78 d'entre eux. Or une grande majorité, 48, demeurent en fait avec leur nourrice qui se déclare aussi être leur mère (exactement la situation que les institutions cherchaient à combattre), 18 autres retrouvent leur mère, 8 leur père et 4 leurs grands-parents ${ }^{19}$. La menace d'un contrat de travail qui " retiendrait " l'enfant trouvé jusqu'à la majorité a peut-être fait réagir les parents à ce moment précis. Sans prendre en considération ici l'aspect éthique de la question, il est évident que toutes ces familles ont réussi ainsi à résoudre la difficulté économique de la charge de l'éducation de l'enfant jusqu'à 7 ans. D'autres motifs d'abandon ont pu exister, bien entendu, mais le moment de la récupération de l'enfant est, à lui seul, significatif. Du reste, des histoires de retrouvailles marquées par l'insuccès, qui finissent par le retour à la Misericórdia ${ }^{20}$, ou par un nouvel abandon, non plus à l'hôpital mais dans les rues de la ville ${ }^{21}$, vont aussi dans le sens d'une motivation d'ordre économique dans la décision de récupération de l'enfant, désormais force de travail. L'illégitimité apparaît comme la cause de l'abandon dans un seul cas, largement documenté : celui d'António, abandonné en avril $1724^{22}$, et rendu à son père le 6 novembre $1733^{23}$.

Finalement, les enfants du troisième groupe, ceux qui ont bénéficié d'un contrat de travail signé sous l'égide de la Misericórdia d'Évora, nous sont mieux connus.

\section{LES ASPECTS MATÉRIELS DES CONTRATS DE TRAVAIL}

La première interrogation qui surgit au regard des 242 contrats de travail (130 jeunes filles et 112 garçons) provient de la comparaison implicite avec les deux 
autres groupes. Pourquoi les nourrices les ont-elles rejetés ? Pourquoi la famille n'est-elle pas apparue pour les réclamer ? Comme les deux groupes précédents, ils ont aussi eu majoritairement une seule nourrice pour toute la période et, comme les autres, ils ont été abandonnés à l'hôpital ou à la porte d'une nourrice et non dans n'importe quel lieu de la ville. Mais peu d'autres informations sont disponibles. Les documents restent silencieux quant au destin des enfants entre le dernier salaire reçu par la nourrice et la date de passation du premier contrat par exemple, or plusieurs années pouvaient s'écouler entre les deux événements. Qu'advenait-il de ces enfants durant cet intervalle ? Retournaient-ils à l'hôpital qui les avait accueillis quand ils étaient bébés ? Étaient-ils internés à l'hospice pour les pauvres, comme certains documents l'attestent pour le XIXe siècle ? Restaient-ils chez leur nourrice, la servant en échange du toit et du couvert ? Cette hypothèse est le plus souvent mentionnée, mais sans doute ne s'agit-il pas d'une règle absolue.

Les limites chronologiques de cette pratique de contrats ne sont pas non plus expliquées mais rien ne suggère qu'elle ait commencé avant 1669 et qu'elle se soit prolongée au-delà de 1788. Si de plus grandes préoccupations légalistes peuvent avoir justifié le début de sa réalisation, il convient surtout de souligner qu'elle apparait au moment de la réorganisation des structures de charité et d'assistance dans la ville avec la création du Colégio dos Órfāos précédemment mentionné. À cette époque, le Recolhimento da Piedade, le Colégio dos Órfãos et la Misericórdia, en tant que gestionnaire des enfants trouvés, avaient le même projet éducatif, dirigé vers des réalités sociales très semblables, même s'ils relevaient de statuts légaux différents. Fondé dans le but d'héberger des garçons orphelins et abandonnés, le Colégio dos Orfãos réduisait pratiquement son domaine d'intervention aux orphelins de la ville, âgés de plus de 7 ans, dont le père et la mère étaient identifiés (Guedes, 2000), alors que la Piedade remplissait un rôle identique pour les filles des pauvres, laissant à la Misericórdia le soin de s'occuper de l'avenir des enfants trouvés. Sous la tutelle des trois institutions se dessinait un même destin professionnel pour les enfants : les filles seraient exclusivement employées dans des services domestiques, les garçons dans les métiers de l'artisanat. La voie des études était interdite aux orphelins accueillis au Colégio dos Inocentes, leur statut était sur ce point très clair et révèle l'esprit qui animait ces institutions. Gérées par les mêmes hommes (Pardal, 2004), elles se voyaient imposer les mêmes règles de fonctionnement. Mais ce n'est là qu'une partie de la question. De fait, la Piedade et la Misericórdia sont devenues une sorte de dépôt où les classes moyennes et les milieux modestes de la population venaient recruter leur main-d'œuvre.

Dans le cas qui nous intéresse au premier chef, celui de la Misericórdia, l'appartenance sociale des maîtres - toujours désignés par le terme de " tuteur ", un statut juridique qu'ils assumaient - illustre bien l'affirmation précédente. Parmi les tuteurs dont la profession a été enregistrée, les artisans, les agriculteurs et les professions liées aux travaux agricoles, et enfin quelques marchands, constituent la presque totalité du spectre socioprofessionnel. Un nombre significatif de femmes, surtout des veuves, et une présence très restreinte des membres de l'Église et de la petite 
élite locale, complètent le tableau ${ }^{24}$. Même sous cet angle, le cas d'Andresa, l'enfant dont nous évoquions le parcours au tout début de ce texte, est vraiment exceptionnel. Dans une ville éminemment aristocratique, peuplée de grands propriétaires terriens et d'un clergé trop nombreux (pour reprendre le témoignage de Manuel Severim de Faria), constater leur absence presque totale parmi les tuteurs des enfants trouvés ne peut que suggérer une attitude délibérée, le refus d'employer ces enfants, qui parfois pouvaient être les leurs.

Ces contrats, qui leur offraient la possibilité de travailler chez des cordiers, des maçons, des forgerons, des prêtres et beaucoup d'autres, permettaient aux enfants une première insertion dans le monde du travail mais aussi une première expérience d'intégration sociale. Leur capacité d'autosubsistance était aussi implicitement reconnue. En sortant de chez leur nourrice, ils s'ouvraient au monde, même si celui-ci demeurait un peu restreint. À l'inverse de leurs homologues simplement " adoptés ", ils disposaient, du moins théoriquement, d'une plus grande capacité d'intervention sur leur proche avenir.

La durée des liens contractuels et le montant des salaires à payer se sont maintenus à un niveau relativement stable au cours de la période étudiée ; ils étaient identiques pour les deux sexes. L'âge de l'enfant trouvé au moment de la signature du contrat est important, car il s'agissait du seul élément susceptible d'introduire quelques modifications concernant la durée du contrat ou le salaire à verser.

L'enfant était disponible pour le marché du travail à l'âge de 7 ans même si le premier contrat pouvait être établi quelques années plus tard. L’objectif des gestionnaires de la Misericórdia semble avoir été de fournir aux jeunes, jusqu’à l'âge de 18 ans, une certaine stabilité "familiale ", sous tutelle masculine, même si de rares contrats ont été pensés en fonction des 20 ans de l'enfant trouvé. En pratique, les contrats pouvaient avoir une durée entre 8 et 10 ans - ces derniers étant les plus fréquents ${ }^{25}$-, et se prolonger sur des périodes plus longues dans des situations particulières. Cependant, dans tous les cas, les responsabilités matérielles des tuteurs envers les enfants jusqu'à l'âge de 12 ans (rarement 11 ans) se limitaient à l'alimentation, à l'habillement et au logement ${ }^{26}$. Ce n'est qu'après cet âge qu'ils commençaient à payer un salaire, qui était remis une fois le contrat terminé (à moins que des situations exceptionnelles n'aient provoqué son annulation). Si la responsabilité de l'interruption du contrat en revenait au tuteur, les salaires versés devaient être doublés ${ }^{27}$. Les 1000 réis annuels auxquels les jeunes avaient droit, pouvaient prendre la forme d'une parure de lit dans le cas des jeunes filles, si tel était leur désir ${ }^{28}$.

Au début du XVIII siècle le traitement médical des enfants trouvés aux frais des maîtres commence à apparaître parmi les obligations des employeurs. Cependant, la nouvelle clause la plus frappante est celle qui oblige le tuteur à hypothéquer ses biens - ou à trouver un cautionnaire qui le fasse à sa place - afin de garantir le respect de ses engagements. L'inclusion de cette nouvelle garantie paraît motivée par des cas répétés de veuves ne pouvant plus verser le salaire dû, à cause du décès de leur mari. Il fallait certes régler cette injustice, mais le préjudice subi était particulièrement grave car, au moins pour les jeunes filles, ce pécule représentait la dot qui leur 
ouvrait les portes du mariage. Or le revenu perçu était précisément justifié par l'objectif de "prendre état ". Lorsque l'on parvenait à cette issue recherchée, l'enfant trouvée passait directement de la tutelle du maître à celle du mari sans avoir accès à son argent, une fois que l'accord était établi entre les deux hommes ${ }^{29}$.

Le déterminisme assumé de la condition féminine est un élément important de ces documents et c'est sur lui que se fondaient les principales différences contractuelles entre les sexes. Il est évident que la formation domestique et sociale des filles était une plus grande préoccupation que celle des garçons.

Tant qu'elle servait comme domestique, la jeune fille devait être formée selon les valeurs qui feraient d'elle une bonne épouse et toute son éducation devait être orientée en fonction de ce but. Tout d'abord la préservation de l'honneur, c'est-à-dire le maintien de la virginité jusqu'au mariage, une valeur en soi ; tous les contrats obligeaient les maîtres à maintenir les jeunes filles enfermées (" servir des portes vers l'intérieur ") et ne permettaient une sortie dans la rue que si elles étaient accompagnées par les femmes de la maison, et cela dès qu'elles "étaient en danger ", c'est-à-dire à partir de l'âge de 12 ans. Le non-respect de cette règle donnait lieu à l'annulation des contrats et les enfants étaient retirées à leurs tuteurs ${ }^{30}$.

Pour les garçons, le mariage n'était jamais mentionné comme un objectif, à l'inverse de l'apprentissage d'un métier. Ici deux situations différentes pouvaient se présenter : si le tuteur était un artisan, l'enfant n'avait droit à aucun salaire pendant la durée du contrat, en échange le maître s'engageait à lui enseigner son métier pendant cette période. S'il ne le faisait pas, il devait payer un de ses collègues afin qu'il assurât ces fonctions jusqu'à ce que le jeune fût considéré apte à l'exercice du métier concerné ${ }^{31}$. Une prérogative que l'institution semble avoir défendue avec un certain souci car l'on trouve des demandes de prolongation de la durée de l'apprentissage lorsqu'une maladie de l'enfant avait raccourci son temps de formation ${ }^{32}$. Quand le tuteur n'était pas artisan, le garçon recevait son salaire - également à la fin de la période - mais le maître devait lui payer l'apprentissage d'un métier, au choix du jeune garçon ${ }^{33}$, à la fin du contrat.

\section{SUR LE PLAN AFFECTIF}

Mais les contrats de travail étudiés sont bien plus que des documents relativement répétitifs et monotones qui réglementent l'avenir professionnel des enfants trouvés. Les textes eux-mêmes, mais aussi les annotations inscrites en marge au fil des années, dévoilent des attachements, des peurs et des angoisses, des sentiments que le greffier n'a pas enregistrés mais qui pointent derrière certains comportements rapportés. Et aussi parfois, des histoires individuelles que l'on devine très complexes. Les documents sont par exemple prolixes en ce qui concerne les difficultés d'adaptation des enfants trouvés dans leurs foyers d'affectation.

Dans tous les contrats de travail, le maître s'engageait à " bien traiter " les enfants qu'il accueillait chez lui, expression qui recouvrait aussi parfois l'enseignement de la doctrine et des bonnes mœurs ; il s'y ajoutait la promesse de les employer seulement à des " tâches justes et honnêtes ". Même si les contrats ne mentionnaient pas la possibilité d'abandonner les maîtres ${ }^{34}$, on ne relève pas de 
cas où un enfant ait été obligé de retourner dans un foyer d'où il avait fugué ou qu'il avait quitté par mandat de la Misericórdia lorsque les signes de « la mauvaise vie qui lui était donnée " étaient évidents ${ }^{35}$.

Les histoires, nombreuses, de fugues d'enfants concernaient presque exclusivement les jeunes filles ${ }^{36}$. La réclusion à laquelle elles étaient soumises à partir de l'adolescence eut un rôle déterminant dans ce comportement ${ }^{37}$. En règle générale, la solution trouvée - puisque la plupart du temps l'enfant fuyait vers l'hôpital qui l'avait recueillie au moment de l'abandon - consistait à placer la jeune fille dans un autre foyer, souvent celui de confrères de la Misericórdia. Mais l'itinéraire n'était pas toujours aussi simple. Certains cas laissent entrevoir un parcours de prostitution, que l'institution a puni par une tentative de réforme morale. C'est ce qui semble s'être produit pour l'enfant trouvée Josefa - ou pour Maria ${ }^{38}$-, dont l'itinéraire de fugitive se termine par la réclusion au Recolhimento da Madalena, une maison destinée à récupérer les «femmes perdues».

Recueillie par la Misericórdia le 8 octobre 1717, Josefa fut élevée par deux nourrices jusqu'à l'âge de 10 ans. Le 29 octobre 1727 , l'institution signa un contrat de travail pour une durée de huit ans avec Manuel Lopes Coelho, contrat qu'en se réfugiant à l'hôpital l'enfant trouvée ne respecta pas. En mars 1730, la Misericórdia signa un autre contrat, cette fois avec le maçon Bento João et sa femme Juliana Maria, pour 5 ans seulement, car Josefa avait déjà 13 ans. L'enfant s'enfuit une fois de plus vers l'hôpital, jusqu'à ce qu'un nouveau contrat, en mars 1735 , la place chez le marchand João Martins. Cette foisci, la situation s'inversa : c'est son patron qui l'expulsa. Cependant, l'inactivité de
Josefa dura peu de temps puisque le barbier Tomé Freire la prit à son service. Le contrat fut signé pour un an, avec l'indication qu'elle recevrait un salaire de 4000 réis, sauf si, à la fin de la durée déterminée, le barbier jugeait opportun un nouvel ajustement contractuel. Mais après quelques jours seulement, ce nouveau tuteur rendit Josefa à la Misericórdia. Celle-ci se retourna vers la Casa das Convertidas da Madalena, où la jeune femme entra en juin $1735^{39}$.

Dans l'exercice de l'autorité paternelle que la fonction de tuteurs leur conférait, les maîtres pouvaient décider d'enfermer les enfants dans les monastères - comme le fit Lourenço da Costa pour l'enfant trouvée Mónica, en 1690, lorsqu'elle eut $11 \mathrm{ans}^{40}$. Les raisons pour lesquelles la Misericórdia acceptait de pareilles conditions ne sont pas explicites, même si l'acceptation de la paternité illégitime est sous-entendue dans certains exemples. Il en fut du moins ainsi pour Lourenço da Costa $^{41}$. Cependant les souhaits de ce tuteur ne furent pas réalisés car Mónica fut placée comme domestique chez le cordier Nicolau Simóes. Genoveva, abandonnée en 1719, eut un autre parcours qui semble démontrer l'inutilité des vocations forcées ${ }^{42}$. Rejetée successivement par plusieurs tuteurs, et finissant par s'enfuir à l'âge de 10 ans du domicile du seul qui l'avait gardée, elle fut envoyée au couvent de S. Bento de Cástris, par ordre de la Misericórdia. À 18 ans, nous la retrouvons chez João Correia, apothicaire de la ville, sans que soient indiquées les raisons de sa sortie du couvent.

\section{QUELQUES CONCLUSIONS}

Placés sous la responsabilité des municipalités ou des Misericordias, ou des deux, les enfants abandonnés avaient en 
moyenne une vie très brève au Portugal comme dans les autres pays européens ${ }^{43}$. La prise de conscience de ce niveau de mortalité très élevé par l'ensemble de la société fut lent et entraîna seulement quelques rares résultats lorsque des réformateurs sociaux traitèrent de cette question à partir d'un point de vue économique ${ }^{44}$. Non seulement à partir de considérations macro économiques comme la richesse des Nations mais aussi à partir de l'observation de la dégradation des conditions de vie des pauvres qui, depuis la seconde moitié du XVIII ${ }^{\mathrm{e}}$ siècle, sont considérées comme le vivier qui alimente les tours ${ }^{45}$.

En termes politiques, la tolérance officielle du phénomène de l'abandon - qui traverse toute l'Époque moderne portugaise - est suivie d'une répression violente, menée par la bourgeoisie libérale, responsable de la laïcisation de l'assistance aux enfants trouvés (loi du 19 septembre 1836) puis de la fermeture des tours, à partir de la fin des années 1860 (voir Lopes, 2000, 170-176). À partir du début du XIXe siècle, des discours moralisateurs et exaltés contre l'abandon des enfants apparaissent puis se répandent au fur et à mesure que s'accumulent les premiers chiffres nationaux de l'abandon qui montrent que les réformes engagées n'aboutissent pas aux résultats escomptés. D'une estimation de 10000 enfants, par an et en moyenne, abandonnés par leurs parents pendant les années 1820 (Pinto, 1828), l'on passe à un chiffre de 33885 enfants abandonnés dans tout le pays en 1851 pour une population totale évaluée à 3829108 habitants (Almanach..., 1855, 649). Ces chiffres constituaient autant d'indices alarmants qui alimentaient les nombreuses discussions qui divisaient les penseurs sociaux sur un sujet aussi délicat : l'abandon continuait à signifier la peine capitale pour les enfants dont il était le destin. Si les proportions de décédés à cette époque étaient certainement plus faibles que les 98,3\% enregistrés à Setúbal entre 1679 et 1717 , ou les $80,5 \%$ à Évora entre 1650 et 1837 , elles étaient cependant encore considérables.

Dans des conditions de si forte mortalité, l'analyse du destin des enfants survivants à l'époque moderne relève quelque peu d'un exercice rhétorique mais il permet de montrer les limites des considérations sur la circulation des enfants et des propos sur la main-d'œuvre gratuite qu'ils constituaient. Cela ne signifie pas que de tels cas n'existaient pas, mais il ne s'agissait que de situations tout à fait exceptionnelles. À Évora, sur deux siècles, comme nous l'avons souligné, seuls 2001 des 16055 enfants abandonnés survécurent suffisamment longtemps pour que se pose la question de leur travail.

Compte tenu d'une survie particulièrement faible, et en excluant ceux repris par leur famille ou gardés par leur nourrice, les enfants qui ont bénéficié de quelques possibilités d'intégration sociale avec l'appui des Misericórdias ont été en nombre nécessairement très limité. Les 242 contrats de travail signés en 120 ans par la Misericórdia de Évora correspondent, en moyenne, à deux enfants placés sur le marché du travail de cette grande ville chaque année (un chiffre qui surestime les valeurs du XVII siècle - où la moyenne est de moins d'un contrat par an - et se font plus proches de la réalité à mesure que l'on avance dans le XVIII siècle). Peut-on en déduire que le projet de la Misericórdia a complètement échoué ? Une évaluation ne saurait se limiter à des aspects seulement quantitatifs et matériels.

Il est certain qu'à Évora l'abandon semble avoir constitué une variable d'ajustement afin de maintenir la compatibilité 
entre la taille des foyers et les ressources économiques disponibles. Cette hypothèse se fonde sur deux éléments principaux. En premier lieu, notre connaissance des institutions et réseaux d'assistance formels existant à Évora et de leur pratique de sélection des familles secourues, permet d'affirmer que les aides accessibles ne pouvaient répondre aux besoins des couples en situation de pénurie prolongée. Cette tension l'a probablement emporté sur d'éventuelles préoccupations de sauvegarde d'hypothétiques codes d'honneurs au moment de décider de l'abandon.

En second lieu, la mortalité considérable des enfants trouvés à Évora comme à Setúbal, deux des plus grands centres urbains du pays à l'époque moderne, n'échappait ni aux élites ni aux habitants. Les nombreux décès qui survenaient au cours des premiers jours, à l'hôpital de $S$. Lázaro (Évora) et de Espírito Santo (Setúbal) ne pouvaient passer inaperçus. En outre, les nourrices habitaient le plus souvent dans la ville, par conséquent dans un espace circonscrit, et tout le voisinage assistait nécessairement à la mort fréquente des nouveaux nourrissons reçus (au point que des procès pour négligence leur étaient intentés). Le destin probable de leur bébé était donc parfaitement connu des parents qui l'abandonnaient, d'autant plus qu'ils vivaient fort probablement en majorité sur place, dans la ville (l'estimation de leur proportion reste toutefois à préciser $)^{46}$.
Finalement, le plus troublant ne résidet-il pas dans l'écart considérable qui sépare les discours, les déclarations, des pratiques ? Ces enfants abandonnés étaient bien identifiés comme vulnérables et les rares qui survivaient étaient en quelque sorte protégés par le contrôle de l'institution lors de leur placement. Mais pendant près de trois siècles les communautés n'ont pas été capables de mobiliser les moyens financiers et humains (en particulier de payer les nourrices) qui auraient permis de réduire l'hécatombe. Comment interpréter une telle passivité ? En sauvant leur âme par le baptême, considérait-on que l'essentiel avait été fait ? La sensibilité à l'égard de l'enfance n'apparaît-elle que très tardivement $\mathrm{du}$ fait de la forte mortalité générale aux jeunes âges ? Pourquoi les élites locales n'ont-elles jamais réussi à mobiliser suffisamment de ressources, alors que les discours sur la croissance souhaitable de la population étaient si nombreux ? Comment expliquer cette longue inertie? Autant de questions qui supposent un retour vers les modes de financement des différentes institutions qui prenaient en charge ces enfants et vers les modalités de prise de décision.

$$
\begin{array}{r}
\text { Laurinda ABREU } \\
\text { Département d'histoire } \\
\text { Palacio de Vimioso, Ap. } 94 \\
\text { Universidade de Evora } \\
7000-820 \text { Evora } \\
\text { Portugal } \\
\text { laurida.abreu@mail.telepac.pt }
\end{array}
$$

\section{NOTES}

1. ADE, (Arquivo Distrital de Évora), Baptismos da $S e ́, \mathrm{n}^{\circ} 21,1653-1660$, fl. 82.

2. Elle a reçu pour ce travail 37000 réis. Voir Engeitados: receita e despesa. Foros, rendas, rendas de pão, ordenados, no 991 a 998, (1658-1666).
3. Livro dos assentos das pessoas que leuão para seu seruiço os engeitados deppois de idade de sete annos com obrigação de darem conta delles a esta meza. 1669/1726, no 1080 , fl. 7.

4. Livro das Lembranças, fol. $193 \mathrm{v}$. 
5. Livro dos assentos das pessoas que leuão para seu seruiço os engeitados, $\mathrm{n}^{\circ} 1080$, fl. $8 \mathrm{v}$.

6. Ibidem, fl. 13.

7. En marge de ce travail reste l'étude de l'appui que la Misericórdia donnait aux orphelins. Il s’agit d'une base de données encore en construction, bien plus limitée du point de vue chronologique - ces données ne sont disponibles que de 1632 à 1712 - mais significative du point de vue numérique, puisqu'elle identifie 355 orphelins dans la période 1632-1682. La logique de fonctionnement de ce service d'assistance était différente de celle des enfants trouvés puisque dans la majorité des cas la prime attribuée au conjoint survivant pour aider à l'éducation de l'enfant était temporaire. Lorsque l'enfant avait perdu ces deux parents, une nourrice était engagée pour compléter son éducation jusqu'à l'âge de 7 ans selon des modalités identiques à celles des enfants trouvés.

8. Notons le sous-enregistrement des années 1680-1692.

9. L'abandon des nouveau-nés ne peut se réduire à sa dimension économique. En outre, les villes constituaient, du fait de la concentration des ressources et de la capacité politique à les mobiliser, les lieux principaux d'abandon des enfants dans les communautés les plus pauvres.

10. Les dépouillements sytématiques en cours permettront de préciser le niveau global d'illégitimité. À Porto, la proportion passée de $8 \%$ des baptêmes pour la décennie 1620-29 à 13,6\% pour 1680-1689. Les recherches qualitatives déjà réalisées sur Évora indiquent la faiblesse de la discrimination sociale à l'égard des enfants illégitimes.

11. Les données économiques et les séries de prix des céréales ont été rassemblées par Rui Manuel Leitão da Silva Santos (1995).

12. Il ne s'agit pas ici d'étudier de façon détaillée la mortalité des enfants abandonnés comme ont pu le faire par exemple Jean-Pierre Bardet, Corinne Martin-Dufour et Jacques Renard (1994).

13. Nous ne trouvons sur cette période aucune règle définie sur le temps pendant lequel les nourrices devaient allaiter et cette étude n'a pas été faite à partir des registres de salaires.

14. Selon le rapport élaboré à l'époque, l'hôpital avait accueilli 529 enfants entre le 18 septembre
1811 et le 14 mai 1816 , dont 414 ont fini par mourir. Le fait que la nourrice résidant à l'hôpital ne pouvait pas allaiter les enfants qui arrivaient, est désigné comme l'une des raisons pour cette situation. À la suite de cette investigation, la nourrice a été renvoyée ainsi que toute l'administration de la Misericórdia. Parmi les mesures appliquées, on note une préoccupation au sujet des nourrices - non seulement durant le processus de sélection mais aussi au niveau de l'amélioration des conditions de paiement. (Voir ADE, Autos de Revista dos Expostos para que procedeu em auto de correição o doutor provedor destas comarcas, livre 497). La perception de la relation fonctionnelle entre l'allaitement et le taux de mortalité des enfants trouvés, est évidente dans ce document, illustrant pour le Portugal, ce que Carlo Corsini (1991) a analysé pour Florence.

15. Livro das Lembranças, no $1709-1$ 719, Fl. 204 - 25 de Abril de 1718.

16. Les questions de caractère économique et toute la problématique liée aux nourrices ne seront pas abordées dans le cadre de cet article.

17. Legados Pios - Santa Casa da Misericórdia d'Évora, Évora, Typ. da Casa Pia, 1882, p. 1.

18. Sur le sujet, voir (Sá, 1995, 305-308).

19. Ana, Joana, João, Francisco et Micaela, ont été récupérés au moment précis de la célébration du contrat. (Livro dos assentos das pessoas que leuão para seu seruiço os engeitados, $\mathrm{n}^{\circ} 1080$, fl. $14 \mathrm{v}$ (João); fl. 70v (Ana); livre $\mathrm{n}^{\circ} 1$ 081, 1726-1788, fl. 4v (Micaela); fl. 8 (Joana); fl. 67v (Francisco). Une situation identique est décrite par Jean-Pierre Gutton (2003, 929-939), ce qui montre que les parents connaissaient bien leur enfant, bien que l'on veillât à conserver l'anonymat des enfants trouvés.

20. Dans le cas de João, le premier contrat ayant échoué à la demande de la mère, il finit par retourner à la Misericórdia qui l'emploie comme domestique d'un archidiacre de la cathédrale (Livro dos assentos das pessoas que leuão para seu seruiço os engeitados, $\mathrm{n}^{\circ} 1080$, fl. 14v).

21. Comme cela est arrivé à Francisco : remis à

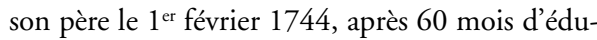
cation, il fut retrouvé mendiant le 29 avril suivant, et recueilli par son ancienne nourrice, avec un contrat de travail de neuf ans.

22. Livro dos assentos das pessoas que leuão para seu seruiço os engeitados, no 1081 , fls. 20-27. 
23. Le père connaît bien l'histoire de son fils, qu'il décrit depuis le jour de l'abandon jusqu’à la signature du contrat de travail. La culpabilisation des grands-parents de l'enfant - issus de l'élite locale - persuade les dirigeants de la Misericórdia de rendre le garçon à son père sous " obligation et à condition de le garder chez lui et de le traiter comme un fils en lui donnant une bonne éducation et un bon traitement faisant en sorte qu'il ne devienne pas un préjudice pour la république ». Une phrase suffisamment claire quant à l'avenir d'António et qui n'était certainement pas celui de la légitimation.

24. Non identifiés : 42 ; femmes : 48 (parmi lesquelles 12 nourrices); diplômés et élite sociale : 26 ; artisans : 70 (21 cordonniers, 11 tailleurs, 8 orfevres) ; prêtres : 21 ; marchands : 9 ; agriculteurs et autres professions : 18 .

25. Exceptionnellement, on trouve référence à des contrats d'une durée de 15 ans ; la mention " ou plus si l'enfant trouvée le désire " est ajoutée sur celui de Josefa (Livro dos assentos das pessoas que leuão para seu seruiço os engeitados, no 1080 , fl. 54v).

26. Dans de rares cas, comme celui de Brásia, les tuteurs vont à la rencontre des enfants avant la fin du processus d'éducation et expriment le désir de les élever selon les principes de la maison qu'ils allaient servir (Livro dos assentos das pessoas que leuão para seu seruiço os engeitados, $\mathrm{n}^{\circ} 1080$, fl. 43v).

27. Ce qui ne se produit pas en cas de situations extraordinaires, comme une maladie prolongée (Dionísia), ou une fugue de l'enfant (Domingos) (Livro dos assentos das pessoas que leuão para seu seruiço os engeitados, livre $\mathrm{n}^{\circ} 1080$, fls. 19-19v (Dionisia), (livre $\mathrm{n}^{\circ} 1081$, fls. 93-94v [Domingos]).

28. Quand la possibilité de choix leur était offerte, l'option pouvait être bien moins utilitaire et la parure de lit habituelle être remplacée par des boucles d'oreille en or (Livro dos assentos das pessoas que leuão para seu seruiço os engeitados, no 1081 , fls. 3-3v).

29. Dans les contrats de ces 242 enfants trouvés, seuls sept jeunes filles et deux garçons sont mentionnés comme étant mariés : les professions des époux renseignent bien sur l'horizon social qui attend les jeunes filles : selliers, tailleurs et infirmiers.
30. Bien que l'institution puisse annuler la décision prise, comme dans le cas de l'enfant trouvée Páscoa, à l'âge de 11 ans. L'annulation du contrat signé en 1673 par le confrère, le capitaine Bartolomeu Ramos, fut considérée sans effet parce que la jeune fille aimait travailler chez lui et parce que le maître fit promesse que la situation ne se répéterait pas (Livro dos assentos das pessoas que leuão para seu seruiço os engeitados, no 1080 , fl. 10).

31. Ibidem, fls. 60v-70.

32. Ibidem, fl. 58.

33. Nous avons trouvé un cas, unique, de contrat dans lequel il était prévu que le jeune garçon pourrait choisir un métier selon ses goûts, sauf celui de maçon (Livro dos assentos das pessoas que leuão para seu seruiço os engeitados, no 1081 , fls. 20-27).

34. Livro dos assentos das pessoas que leuão para seu seruiço os engeitados, $\mathrm{n}^{\circ} 1080$, fl. 12.

35. Ibidem, fl. 57v.

36. On ne trouve des renseignements que sur la fugue de deux garçons, déjà adultes. La raison donnée fut le désir de se marier sans l'autorisation de la Misericórdia.

37. À l'inverse de ce qui arrivait en France dans les situations mentionnées par Jean-Pierre Gutton (2003, 933) et Scarlett BeauvaletBoutouyrie (2003, 897-898).

38. Livro dos assentos das pessoas que leuão para seu seruiço os engeitados, $\mathrm{n}^{\circ} 1081$, fl. $43 \mathrm{v}$ et $63 \mathrm{v}$.

39. Ibidem, fl. $5 \mathrm{v}$; fl. 12; fl. 29v. Des cas qui semblent identiques mais qui furent peut-être considérés comme moins graves du point de vue social selon le système de valeur en vigueur, se sont terminés par l'" exil » vers Lisbonne. Ainsi Brízida, en 1707 (Idem, fl. 51 et fl. 57) et Ana Preta (Idem, fl. 56v) en 1712. Le placement des enfants trouvées au Recolhimento da Piedade - la maison qui accueillait les jeunes filles pauvres (pour y attendre un contrat de travail ou une dot leur permettant de se marier) - fut moins fréquent qu'on aurait pu l'imaginer.

40. Livro dos assentos das pessoas que leuão para seu seruiço os engeitados, $\mathrm{n}^{\circ} 1080$, fls. $25 \mathrm{v}-27 \mathrm{v}$.

41. Livro das lembranças da mesa, fl. 178.

42. Livro dos assentos das pessoas que leuão para seu seruiço os engeitados, no 1081 , fl. $91 \mathrm{v}$. 
43. Ce qui n'est pas un phénomène exclusivement portugais. Sur la réalité française, voir la synthèse élaborée par Scarlett BeauvaletBoutouyrie (1999, 245-255 et surtout 252-255).

44. Parmi les principaux réformateurs sociaux, on peut citer Manuel Severim de Faria, Duarte Ribeiro de Macedo et Pina Manique.Tous leurs discours aboutirent à la loi du 10 mai 1783 , décidant que toute municipalité dépourvue d'hôpital des enfants abandonnés devait en ouvrir un.
45. La législation $\mathrm{du} X V I^{\mathrm{e}}$ siècle ne mentionne jamais l'exitence de tours. Ils paraissent se répandre au cours du XVII ${ }^{e}$ siècle et certaines villes, comme Évora, ne les installent qu'à la fin du XVIII ${ }^{e}$ siècle, à la suite d'une décision royale.

46. Sur cette question, voir (Pérez Moreda, 1996 34). À Évora, le fait que parmi tous les enfants trouvés rendus à leurs familles, on ne recense qu'un seul cas où l'enfant quitte la ville pour vivre avec sa mère à la campagne plaide aussi dans le sens d'une forte concentration des mères dans la ville.

\section{RÉFÉRENCES BIBLIOGRAPHIQUES}

Abreu, Laurinda (2003), “The Évora Foundlings between the $16^{\text {th }}$ and the $19^{\text {th }}$ Century: the Portuguese Public Welfare System under Analysis", Dynamis. Acta Hispânica ad Medicinae Scientiarumque Historiam Illustrandam, Granada, vol. 23, 37-60.

Almanach de Portugal para o ano de 1855 (1854).

BARDET, Jean-Pierre, MARTIN-Dufour, Corinne et Renard, Jacques (1994), " La mort des enfants trouvés, un drame en deux actes», Annales de Démographie Historique, 135-150.

Beauvalet-Boutouyrie, Scarlett (1999), La démographie de l'époque moderne, Paris, Belin.

Beauvalet-Boutouyrie, Scarlett (2003), "Les enfants de la Salpêtrière au XVIII siècle ", 883-901, in Lorsque l'enfant grandit. Entre dépendance et autonomie, JeanPierre Bardet, Jean-Noël Luc, Isabelle Robin-Romero et Catherine Rollet (dir.), Paris, Presses de l'Université de ParisSorbonne.

Brunet, Guy, Bideau, Alain, Rappalini, Emmanuelle, (2003), "Grandir sans famille : enfants et adolescents assistés en Haute-Savoie au XIX" siècle », 901-915, in Lorsque l'enfant grandit. Entre dépendance et autonomie, Jean-Pierre Bardet, JeanNoël Luc, Isabelle Robin-Romero et Catherine Rollet (dir.), Paris, Presses de l'Université de Paris-Sorbonne.
Corsini, Carlo A. (1991), "Breastfeeding, Fertility and Infant Mortality: Lessons from the Archives of the Florence Spedale degli Innocenti”, Historical Perspectives on Breastfeeding, Florence, Unicef, 63-85.

Guedes, Ana Isabel Marques (2000), Les enfants orphelins. Éducation et Assistance. Les Colégios de Meninos Órfãos : Évora, Porto et Braga (XVII $\left.-X I X^{e}\right)$, Florence, Institut Universitaire de Florence.

GutTON, Jean-Pierre (2003), " L'insertion sociale des enfants recueillis par la Charité de Lyon au XVIII ${ }^{\mathrm{e}}$ siècle. Un bilan provisoire ", 929- 939, in Lorsque l'enfant grandit. Entre dépendance et autonomie, Jean-Pierre Bardet, Jean-Noël Luc, Isabelle Robin-Romero et Catherine Rollet (dir.), Paris, Presses de l'Université de Paris-Sorbonne.

LOPES, Maria Antónia (2000), Pobreza, Assistência e Controlo Social. Coimbra (1750-1850), vol. I, Viseu, Palimage.

PARDAL, Rute (2004), "O relacionamento do Arcebispado com a Misericórdia de Évora entre 1552 e 1643”, 225-237, in Igreja, caridade e assistência na Peninsula Ibérica (sécs. XVI-XVIII), Laurinda Abreu (dir.), Lisboa, Edições Colibri/Cidehus.

PéreZ MoredA, Vicente (1996), "Infancia abandonada e ilegitimidad en la historia de las poblaciones ibéricas", Expostos e ilegitimos na realidade ibérica. Actas do III congresso da $A D E H$, vol. 3, Porto, Afrontamento. 
PINTO, António Joaquim de Gouveia (1828), Exame critico e histórico sobre os direitos estabelecidos pela legislação antiga e moderna, tanto pátria como subsidiária, e das naçôes mais vizinhas e cultas, relativamente aos expostos e engeitados, Lisboa, Typografia da Academia Real das Ciências.

SÁ, Isabel dos Guimarães (1995), $A$ circulação de crianças na Europa do Sul: o caso dos expostos do Porto no século XVIII, Lisboa, FCG/JNICT.

SANTOS, Rui Manuel Leitão da Silva (1995), Celeiro de Portugal algum dia. Crescimento e crises agrárias na região de Évora. $1595-$ 1850, Lisboa.

SousA, Fernando Alberto Pereira de (1979), A população portuguesa nos inícios do século $X I X$, Porto, Faculdade de Letras.

\section{RÉSUMÉ}

Au Portugal, dès 1514, la législation royale encadre la protection des enfants abandonnés, pris très souvent en charge à partir de la seconde moitié du XVI e siècle, ainsi que les hôpitaux, par les municipalités et les Misericórdias et cela jusqu'en 1836. L'étude systématique effectuée sur la ville d'Évora permet de mettre en évidence l'énorme mortalité des enfants abandonnés (mortalité infantile de 734 pour mille, probabilité d'atteindre l'âge de 7 ans de 150 pour mille), ainsi qu'une corrélation entre le mouvement des abandons et l'évolution des prix des céréales lors des crises, ce qui conduit l'auteur à plaider en faveur d'une pratique d'abandon qui entrerait dans un schéma de régulation de la dimension des familles, même légitimes. L. Abreu suit ensuite le destin de cette petite proportion d'enfants atteignant leur septième anniversaire et placés par la Misericordia (dans la famille de la nourrice $(60 \%)$ mais aussi sur le marché du travail (23\%) ou repris par leur famille (10\%).

L'auteur analyse enfin le développement du discours des élites qui organisent la protection des enfants abandonnés et souligne la distance qui les sépare des réalités : les ressources afin de faire baisser leur mortalité n'ont jamais été mobilisées

\section{SUMMARY}

In Portugal, as soon as 1514, the king's law has framed the protection of abandoned children who were mostly taken into care, starting from the second half of the XVI ${ }^{\text {th }}$ century, as well as hospitals, by the municipalities and the Misericordias - until 1836. A systematic research on the city of Evora allows to bring to the fore the huge mortality among abandoned children (infant mortality: 734/1000; life time expectation up to 7 years: $150 / 1000)$, together with correlated trends between the evolution of abandonment and that of grains prices in times of crises, which induces the author to argue in favour of a practice of abandonment which would fit into a scheme of family size regulation - even of lawful families. L. Abreu then follows the destiny of that small proportion of children having reached their seventh birthday and placed by the Misericordia (in the nurse's family, $60 \%$ - but also on the labour market, $23 \%$ - or taken back by their family - 10\%).

Finally the author analyses the development of the discourse of the elites in charge of organising the protection of abandoned children, and underlines the gap between discourse. 
07_abreu.qxp 28/06/2006 07:50 Page 184

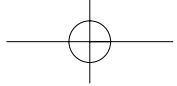

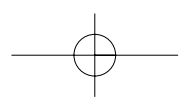

